\title{
DIVERSIDAD GENÉTICA DE 12 LOCIMICROSATELITALES UTILIZADOS EN PRUEBAS DE PATERNIDAD EQUINA EN CHILE
}

\author{
GENETIC DIVERSITY ANALYSIS IN 12 MICROSATELLITE LOCI, USED IN EQUINE \\ PATERNITY TEST IN CHILE
}

Paredes, M. ${ }^{1 *}$, M.C. Norambuena ${ }^{2}$ y B. Molina ${ }^{1}$

${ }^{1}$ Departamento de Ciencias Básicas. Universidad de La Frontera. Av. Francisco Salazar 01145. Temuco. Chile.

${ }^{2}$ Departamento de Ciencias Animales. Pontificia Universidad Católica. Av. Vicuña Mackenna 4860. Santiago. Chile.

*Fono-fax: 56-45-325224. E-mail: mparedes@ufro.cl

\section{Palabras ClaVe ADICIONALES}

Caballo chileno.

\section{RESUMEN}

Se investigó la diversidad genética de 12 loci microsatelitales (HMS2, HMS3, HMS6, HMS7, HTG4, HTG6, AHT4, AHT5, VHL20, ASB2, HTG7y HTG10) utilizados en pruebas de filiación equina en Chile en una muestra poblacional de 45 caballos Criollos chilenos por medio del contenido de información polimórfica, heterocigosis y exceso o déficit de heterocigotos. Además, se estimó la probabilidad de exclusión de paternidad por locus y general considerando los 12 loci. Los resultados indican que la variabilidad genética de los microsatélites analizados es significativamente elevada en la población estudiada. Todos los loci analizados resultaron polimórficos. El número de alelos por locus, varió entre 4 y 10. La heterocigosis esperada promedio $(\mathrm{He})$, considerando todos los loci, fue de 0,76 , con un rango que osciló entre 0,52 y 0,88 . El promedio de la heterocigosis observada (Ho) sobre el conjunto de todos los marcadores fue de 0,65 . La probabilidad de exclusión de paternidad por locus osciló entre 14 a $59 \%$. Al considerar los 12 loci en conjunto, la exclusión de paternidad se puede atribuir con $99,7 \%$ de certeza.

\section{SUMMARY}

The genetic diversity of equine microsatellite loci HMS2, HMS3, HMS6, HMS7, HTG4, HTG6, AHT4, AHT5, VHL20, ASB2, HTG7 and HTG10

Recibido: 1-3-07. Aceptado: 17-9-08.
AdDITIONAL KEYWORDS

Chilean horse.

was investigated in a population of Chilean Creole horses by means of parameters such as: the polymorphic information content, heterocigocity and excess or deficit of heterocigotes. In addition, the probability of paternity exclusion was considered. The results indicated that the genetic diversity of these markers was significantly high in the studied population. All markers analyzed were polymorphic. The number of alleles per locus, varied between 4 (HMS10) to 10 (HTG10). The expected average heterocigocity $(\mathrm{He})$ considering all loci simultaneously, was 0.76 ranging from 0.52 to 0.88 . The average observed heterocigocity $(\mathrm{Ho})$ for all the markers was 0.65 . The paternity exclusion probability per locus, oscillated between 14 and $59 \%$. The power paternity exclusion considering the 12 loci was $99,7 \%$.

\section{INTRODUCCIÓN}

El tipo de grupo sanguíneo y los polimorfismos bioquímicos se han utilizado para la certificación de filiación tanto en humanos como animales de interés pecuario (De Andrés Cara, 1982; Bowling y Clark, 1985; Oltra et al., 1993; Kelly et al., 2002). Sin embargo, la tendencia actual es la utilización de diversos tipos de marcadores de ADN (Giovambattista et al., 2001; Arangu- 
ren-Méndez et al., 2001). Entre los marcadores moleculares de mayor utilidad se encuentran los microsatélites, los cuales están formados por secuencias nucleotídicas de entre dos a seis pares de bases que se repiten un número variable de veces dentro de una disposición en tandem flanqueada por secuencias no repetitivas (Tautz, 1989). El alto nivel de variación en el número de repeticiones genera una alta diversidad alélica, encontrándose entre 2 a 25 alelos por locus, con niveles de heterocigosis que fluctúan entre el 30 y 90\% (Waldbieser y Bosworth, 1997; Hancock, 1999). Estas características, además de la herencia mendeliana y expresión codominante de los microsatélites (Chambers y MacAvoy, 2000), permiten su utilización en la resolución de la filiación con una probabilidad cercana al 100\% (Aranguren-Mendez et al., 2001).

Los loci microsatelitales equinos, HMS2, HMS3, HMS6, HMS7 (Guerin et al., 1994), HTG4, HTG6 (Ellegren et al., 1992), AHT4, AHT5 (Binns et al., 1995 ), VHL20 (Van Haeringen et al., 1994), ASB2 (Breen et al., 1997), HTG7, HTG10 (Marklund et al., 1994), han sido validados en cuanto a sus características hereditarias, diversidad alélica, polimorfismo y rango de tamaño de sus alelos, como marcadores adecuados para pruebas de filiación equina (Ellegren et al., 1992; Bowling et al., 1997).

La efectividad de estos marcadores en pruebas de filiación requiere conocer los parámetros de diversidad genética específicos de la población, lo cual permite asignar parentesco genético con mayor exactitud (Jamieson y Taylor, 1997). El objetivo de este trabajo fue evaluar la diversidad genética de estos marcadores en caballos Criollos chilenos por medio de parámetros estimativos como, el contenido de información polimórfica (Botstein et al., 1980), la heterocigosis (Ott, 1992) y el exceso o déficit de heterocigotos y determinar la probabilidad de exclusión de paternidad por locus y general considerando los 12 loci, utilizados de acuerdo a Jamieson y Taylor, (1997).

\section{MATERIAL Y MÉTODOS}

Se analizó ADN extraído de muestras de pelo de 45 caballos procedentes de diferentes planteles ubicados entre la VIII y X región del sur de Chile, inscritos en los registros genealógicos de la raza Caballo Chileno del Servicio Nacional de Agricultura.

El ADN se extrajo a partir de bulbos pilosos obtenidos según la metodología descrita por Suenaga y Nakamura, (2005). Para esto, se introdujeron cinco pelos en un tubo Eppendorf de 1,5 ml, cuidando que las raíces quedaran ubicadas en el fondo del tubo, luego se agregaron $150 \mu \mathrm{l}$ de Chelex$1005 \%$ (Bio Rad, Richmond, USA) y $10 \mu \mathrm{l}$ proteinasa $\mathrm{K}(10 \mathrm{mg} / \mathrm{ml})$ y se incubó a $56^{\circ} \mathrm{C}$ por $12 \mathrm{~h}$. Posteriormente la mezcla se agitó en vortex por $10 \mathrm{~s}$ y se calentó luego a $100^{\circ} \mathrm{C}$ por $8 \mathrm{~min}$. Luego, el tubo se agitó por $10 \mathrm{~s}$ en vortex y finalmente se centrifugó a $10000 \mathrm{~g}$. Para la reacción de PCR se tomaron directamente $15 \mu \mathrm{l}$ del sobrenadante y se mezclaron con $5 \mu$ lde tampón para PCR 10x (Tris- $\mathrm{HCl}$ $200 \mathrm{mM}$, pH 8,4 KCl $500 \mathrm{mM}$ ), $2 \mu 1$ de $\mathrm{MgCl}_{2}$ $(50 \mathrm{mM}), 2 \mu 1$ de una mezcla de dNTPs $(10$ $\mathrm{mM}$ cada uno), $1 \mu \mathrm{l}$ de cada cebador (500 $\mathrm{pmol} / \mu \mathrm{l}$, cada uno) $0,5 \mu \mathrm{l}$ de Taq polimerasa, $5 \mathrm{U} / \mu \mathrm{l}$ (Promega), más agua estéril, hasta completar $50 \mu \mathrm{l}$ de volumen final de reacción por tubo. Cada locus fue amplificado en forma individual utilizando un termociclador Perkin Elmer, Gene Amp PCR System 2400, con termocupla. Las condiciones térmicas de amplificación se programaron con una denaturación inicial a $94^{\circ} \mathrm{C}$ por 3 min., seguida de 30 ciclos de amplificación, cada uno consistente en denaturación a $94^{\circ} \mathrm{C}$ por $40 \mathrm{~s}$, hibridación a $60^{\circ} \mathrm{C}$ por $30 \mathrm{~s}$ y extensión a $72^{\circ} \mathrm{C}$ por $35 \mathrm{~s}$. Luego de concluidos los 30 ciclos se programó una extensión final a $72^{\circ} \mathrm{C}$ por $10 \mathrm{~min}$.

Los productos de amplificación se analizaron por medio de electroforesis en gel de poliacrilamida de alta resolución utilizando el sistema comercial Spreadex Gel Kit 70-250 (Elchrom Scientific). Los geles se prepararon de acuerdo a las instrucciones del fabri- 


\section{ANÁLISIS DE PATERNIDAD EN CABALLO CHILENO}

cante. La electroforesis se realizó en una cámara de electroforesis MiniProteam II (BioRad) a $200 \mathrm{~V}$ y $35 \mathrm{~mA}$ por una hora. La muestra sometida a electroforesis consistió en una mezcla de $10 \mu \mathrm{l}$ de producto de amplificación y $2 \mu \mathrm{l}$ de tampón de carga (azul de bromofenol 0,25\% p/v, glicerol 30\% v/v disueltos en agua). Posteriormente los marcadores fueron visualizados por exposición del gel (previamente incubado en una solución de bromuro de etidio $0,5 \mathrm{mg} / \mathrm{ml}$ ) a luz ultravioleta. El tamaño de los productos amplificados fue estimado utilizando un estándar de $10 \mathrm{pb}$ (Invitrogen). Los genotipos y tamaños alélicos fueron registrados por conteo directo.

La probabilidad de exclusión de paternidad (Jamieson y Taylor, 1997) fue calculada a partir de las frecuencias alélicas observadas utilizando el programa Cervus 2.0 (Marshall et al., 1998). Este mismo programa se usó para estimar la heterocigosis observada (Ho) y esperada (He), así como el contenido de información polimórfica (CIP). Se determinó, la deficiencia o exceso de heterocigotos por locus con la prueba exacta de Hardy-Weinberg según Raymond y
Rousset (1995a). La significación estadística de estos análisis se obtuvo de acuerdo al método de cadena de Markov (Guo y Thomson, 1992), utilizando el conjunto de programas GENEPOP versión 3.1 (Raymond y Rousset, 1995b).

\section{RESULTADOSYDISCUSIÓN}

Los 12 loci analizados, resultaron polimórficos (tabla I). El número de alelos por locus, varió entre un mínimo de 4 (HTG7) y un máximo de 10 (HMS10, HTG10) con un promedio de 7,6 alelos por locus. Este valor, es similar al descrito en caballos de raza Andaluza (Vega-Pla et al., 2006), pero menor al reportado en otras razas españolas como: Jaca Navarra, Asturcon, Caballo Gallego, Losina, Menorquina, Pottoka y Mallorquina en las cuales se describe un promedio de 10,7 alelos por locus (Marletta et al., 2006). La heterocigosis media esperada (He), considerando todos los loci a la vez fue de 0,76 , mientras que su valor mínimo por locus fue de 0,52 en HTG7 y máximo de 0,88 en HTG10 (tabla II). El promedio de la heterocigosis observada (Ho) sobre el con-

Tabla I. Frecuencias alélicas determinadas para cada uno de los 12 loci analizados en caballo Criollo chileno. (Allelic frequencies in the 12 loci analyzed Chilean Creole horses).

\begin{tabular}{|c|c|c|c|c|c|c|c|c|c|c|c|c|c|c|c|}
\hline \multirow[b]{2}{*}{ Locus } & \multicolumn{15}{|c|}{ alelo } \\
\hline & $D$ & $\mathrm{~F}$ & $\mathrm{G}$ & $\mathrm{H}$ & I & $\mathrm{J}$ & $\mathrm{K}$ & L & $M$ & $\mathrm{~N}$ & $\mathrm{O}$ & $P$ & $Q$ & $\mathrm{R}$ & $S$ \\
\hline VHL20 & - & - & 0,28 & - & - & 0,08 & 0,10 & 0,27 & 0,06 & 0,03 & 0,05 & 0,03 & 0,01 & - & - \\
\hline HTG4 & - & - & - & - & - & 0,30 & 0,10 & 0,25 & 0,10 & 0,11 & - & - & - & - & - \\
\hline AHT4 & - & 0,47 & 0,19 & 0,01 & 0,05 & 0,03 & 0,07 & 0,06 & 0,02 & - & - & - & - & - & - \\
\hline HMS7 & - & - & 0,02 & 0,02 & 0,97 & 0,11 & 0,21 & 0,16 & 0,06 & 0,07 & 0,01 & 0,01 & - & - & - \\
\hline HTG6 & 0,11 & 0,09 & 0,29 & - & - & - & 0,03 & 0,38 & 0,02 & - & - & - & - & - & - \\
\hline AHT5 & - & - & - & 0,17 & 0,17 & 0,11 & 0,14 & 0,14 & 0,11 & 0,13 & - & - & - & - & - \\
\hline HMS6 & - & - & - & - & 0,03 & 0,07 & 0,10 & 0,29 & 0,01 & 0,07 & 0,34 & 0,01 & - & - & 0,01 \\
\hline ASB 2 & - & - & - & - & 0,01 & 0,22 & 0,06 & 0,13 & 0,16 & 0,04 & 0,04 & 0,06 & - & - & - \\
\hline HTG10 & - & - & - & 0,05 & - & 0,09 & 0,17 & 0,16 & 0,07 & 0,13 & 0,11 & 0,09 & 0,01 & 0,05 & - \\
\hline HTG7 & - & - & - & - & - & - & 0,17 & - & - & 0,13 & 0,75 & 0,02 & - & - & - \\
\hline HMS3 & - & - & - & - & 0,09 & - & - & - & 0,02 & 0,01 & 0,19 & 0,27 & 0,29 & 0,07 & 0,02 \\
\hline HMS2 & - & - & - & 0,27 & 0,15 & 0,02 & 0,12 & 0,06 & - & - & - & - & - & 0,11 & 0,02 \\
\hline
\end{tabular}

D a S: alelos microsatelitales descritos para equinos. (-) indica la ausencia del alelo respectivo. 
PAREDES, NORAMBUENAYMOLINA

Tabla II. Parámetros estimativos de diversidad genética determinados en 12 loci microsatelitales en caballo Criollo chileno. (Genetic diversity parameters determined in 12 microsatellite loci analyzed in Chilean Creole horses).

\begin{tabular}{lcccccccc}
\hline Loci & $N^{\circ}$ de alelos & Ho & He & CIP & PE & $F_{\text {is }}$ & DE & P \\
\hline VHL20 & 9 & 0,900 & 0,812 & 0,776 & 0,444 & $-0,112$ & 0,004 & 0,945 \\
HTG4 & 5 & 0,600 & 0,748 & 0,697 & 0,333 & $+0,192$ & 0,004 & 0,071 \\
AHT4 & 8 & 0,600 & 0,659 & 0,622 & 0,261 & $+0,110$ & 0,019 & 0,244 \\
HMS7 & 10 & 0,525 & 0,838 & 0,807 & 0,493 & $+0,341$ & 0,000 & 0,001 \\
HTG6 & 6 & 0,650 & 0,733 & 0,682 & 0,317 & $+0,107$ & 0,011 & 0,093 \\
AHT5 & 7 & 0,725 & 0,849 & 0,817 & 0,501 & $+0,149$ & 0,004 & 0,048 \\
HMS6 & 9 & 0,725 & 0,777 & 0,735 & 0,387 & $+0,176$ & 0,001 & 0,007 \\
ASB2 & 8 & 0,500 & 0,820 & 0,786 & 0,456 & $+0,407$ & 0,000 & 0,000 \\
HTG10 & 10 & 0,825 & 0,883 & 0,858 & 0,586 & $+0,093$ & 0,014 & 0,154 \\
HTG7 & 4 & 0,575 & 0,520 & 0,471 & 0,138 & $-0,099$ & 0,005 & 0,063 \\
HMS3 & 8 & 0,775 & 0,787 & 0,744 & 0,394 & $+0,053$ & 0,015 & 0,241 \\
HMS2 & 7 & 0,500 & 0,784 & 0,745 & 0,396 & $+0,332$ & 0,000 & 0,000 \\
\hline
\end{tabular}

Heterocigosis observada ( $\mathrm{Ho})$, heterocigosis esperada $(\mathrm{He})$, contenido de información polimórfica (CIP), probabilidad de exclusión $(P E)$, deficiencia de heterocigotos $\left(F_{i s}\right)$, desviación estándar $(D E)$ y valor $P(P)$.

junto de todos los marcadores fue de 0,65 , con un mínimo de 0,50 (HMS2, ASB2) y un máximo de 0,82 (HTG10). Cabe destacar, que en la mayoría de las razas equinas europeas (Aberle etal., 2004; Marletta et al., 2006) y norteamericanas (Plante et al., 2007), los niveles promedios de heterocigosis de loci microsatelitales son generalmente superiores a 0,65. Recientemente, Luís et al. (2007) analizaron 12 loci microsatelitales similares a los utilizados en este trabajo en ocho razas sudamericanas, encontrando valores promedios de $\mathrm{He}(0,71$ a 0,75$)$ y $\mathrm{Ho}(0,66$ a 0,76$)$, lo cual es relativamente coincidente con los niveles detectados en la muestra poblacional de caballo Criollo chileno analizada.

El promedio del CIP considerando los 12 loci fue de 0,72 , mientras que el mayor valor encontrado fue de 0,85 (HTG10) y el menor de 0,75 (HMS3). Estos valores indican un elevado valor informativo de los microsatélites analizados lo que coincide con lo informado para caballos portugueses (Luis et al., 2002) o árabes (Gralak et al., 1998).

Se detectó deficiencia de heterocigotos en 5 de los 12 loci analizados (HMS7, AHT4, HMS6, ASB2 y HMS2). Este déficit podría deberse a diversos factores como la presencia de alelos nulos que lleva a la observación de una mayor cantidad de homocigotos (Dakin y Avise, 2004). También podría explicarse por una presión de selección sobre estos loci que podrían estar ligados a alguno de los fenotipos sujetos históricamente a selección artificial en esta raza. Los valores de Fis para locus similares descritos en diversas razas de caballos europeos son en generalmente menores a los detectados en nuestra muestra poblacional, aunque cabe destacar que también se han registrado valores altos en alguno de ellos (HMS2) en razas españolas (Marletta et al., 2006). Es interesante destacar, que el análisis de variabilidad genética del mismo grupo de loci microsatelitales utilizados en este trabajo, realizado en otra raza chilena (caballo Chilote) por Mujica et al. (2005), describe valores de Fis, también relativamente mayores a los descritos en poblaciones de caballos europeos (Vega-Pla et al., 2006; Plante et al., 2007), lo cual es más coincidente con estos resultados, pues también se encontró 3 de 12 loci con exceso de homocigotos, sin embargo esta población de caballos confi- 


\section{ANÁLISIS DE PATERNIDAD EN CABALLO CHILENO}

nada a la isla de Chiloé en el sur de Chile, ha sufrido drásticas reducciones del número poblacional lo que junto a su confinamiento insular podría explicar su moderado nivel de endogamia (Mujica et al., 2005).

No se puede descartar tampoco, el efecto de prácticas reproductivas endogámicas, que no son evitadas por los criadores al utilizar un bajo número de reproductores y posiblemente relacionados por consanguinidad, lo cual no es extraño entre criadores de caballos de razas certificadas en Chile.

La probabilidad de exclusión por locus osciló entre 0,138 (HTG7) y 0,586 (HTG10), mientras que el poder de exclusión total (considerando los 12 loci) resultó ser de 0,997 . Esto significa que la utilización del conjunto de 12 loci microsatelitales analizados en el presente trabajo permite la asignación de paternidad o maternidad con un

\section{BIBLIOGRAFÍA}

Aranguren-Méndez, J., J. Jordana and M. Gómez. 2001. Genetic diversity in Spanish donkey breeds using microsatellite DNA markers. Genet. Sel. Evol., 33: 243-252.

Aberle, K.S., H. Hamann, C. Drögemüller and O. Distl. 2004. Genetic diversity in German draught horse breeds compared with a group of primitive, riding and wild horses by means of microsatellite DNA markers. Anim. Genet., 35: 270-277.

Breen, M., G. Lindgren and M. Binns. 1997. Genetic and physical assignments of equine microsatellites-first integration of anchored markers in horse genome mapping. Mamm. Genome, 8 : 267-273.

Binss, M., N. Holmes, G. Hollimans and A. Scott. 1995. The identifications of polymorphic microsatellite loci in the horse and their use in thoroughbred parentage testing. Brit. Vet. J., 151: 9-15.

Botstein, D., R. White, M. Skolnick and R. Davis. 1980. Construction of a genetic linkage map in man using restriction fragment length polymorphisms. Am. J. Hum. Genet., 32: 314-331.

Bowling, A. and S. Clark. 1985. Blood group and protein polymorphism gene frequencies for seven breeds of horse in the United States.
99,7\% de certeza si utilizamos el índice de paternidad de Jamieson y Taylor (1997), ampliamente utilizado para este tipo de pruebas (Gralak et al., 1998; Giovambattista et al., 2001; Luis et al., 2002).

Los resultados obtenidos permiten afirmar que el alto nivel de variación genética encontrado en los 12 loci microsatelitales analizados hace extremadamente improbable asignar erróneamente paternidad en caballo Criollo chileno, sin embargo es recomendable evaluar a mayor escala, la variabilidad genotípica de estos loci ya que no se puede descartar el efecto de los desequilibrios en 5 de los 12 loci analizados. De confirmarse estos desequilibrios tal vez estos loci deberían ser reemplazados por otros en el panel de microsatélites equinos utilizados para pruebas de filiación en Chile.

Anim. Blood Groups Bi., 16: 93-108.

Bowling, A., M. Eggleston-Stott, G. Byrns, R. Clark, S. Dileannis and E. Wictum. 1997. Validation of microsatellite markers for routine horse parentage testing. Anim. Genet., 28: 247-252.

Chambers, G. and E. MacAvoy. 2000. Microsatellites: consensus and controversy. Comp. Biochem. Phys., 126: 455-475.

Dakin, E. and J.C. Avise. 2004. Microsatellite null alleles in parentage análisis. Heredity, 93: 504509.

De Andrés Cara, D. 1982. Pura Raza Española de caballos: comparación con otras razas mediante sus polimorfismos enzimáticos sanguíneos. Tesis doctoral. Universidad de Córdoba. España.

Ellegren, H., M. Johansson, K. Sandberg and L. Andersson. 1992. Cloning of highly polymorphic microsatellites in the horse. Anim. Genet., 23: 133-142.

Giovambattista, G., M. Ripoli, J. Lirón, M. Kienast, E. Villegas-Castagnaso, F. Dulout y P. PeralGarcía. 2001. Aplicación de las técnicas de polimorfismo de ADN en la resolución de casos de abigeato, identificación individual y determinación de paternidad. Anal. Vet., 21: 5-11. 


\section{PAREDES, NORAMBUENAYMOLINA}

Guerin, G., M. Bertaud and Y. Amigues.1994. Characterization of seven new horse microsatellites. Anim. Genet., 25: 124.

Guo, S. and A. Thompson. 1992. Performing the exact test of Hardy-Weinberg proportion for multiple alleles. Biometrics, 48: 361-72.

Gralak, B., J. Kuryl and C. Niemczewski. 1998. Usefulness of a set of seven microsatellites for parentage control in Arabian Horse and Thoroughbreds in Poland. Anim. Genet., 29: 1-6.

Hancock, J. 1999. Micorsatellites and other simple sequences: genomic context and mutational mechanisms. In. Microsatellites: Evolution and applications. (Goldstein, D.B., Schlötterer, C., eds), Oxford University Press. Oxford, England, p. 1-9.

Jamieson, A. and C. Taylor. 1997. Comparisons of three probability formulae for parentage exclusion. Anim. Genet., 28: 397-400.

Kelly, L., A. Postiglioni and D. Andrés. 2002. Variabilidad genética de los caballos Criollos del Uruguay. Arch. Med. Vet., 34: 13-23.

Luís, C., E. Cothran and M. Oom. 2002. Microsatellites in Portuguese autochthonous horse breed: usefulness for parentage testing. Genet. Mol. Biol., 25: 131-134.

Luís, C., R. Juras, M. Oom and E. Cothran. 2007. Genetic diversity and relationships of Portuguese and other horse breeds based on protein and microsatellite loci variation. Anim. Genet., 38: 20-27.

Marletta, D., I. Tupac-Yupanqui, S. Bordonaro, D. García, A. Guastella, A. Criscione, J. Cañon and S. Dunner. 2006. Analysis of genetic diversity and the determination of relationships among western Mediterranean horse breeds using microsatellite markers. J. Anim. Breed Genet., 123: 315-325.

Marshall, T., J. Slate, L. Kruk and J. Pemberton. 1998. Statistical confidence for likelihood-based paternity inference in natural populations. Mol. Ecol., 7: 639-655.

Marklund, S., H. Ellegren, S. Erikson, K. Sandberg and L. Andersson. 1994. Parentage testing and linkage analysis in the horse using a set of highly polymorphic microsatellites. Anim. Genet., 25: 19-23.

Mujica, F., V. Obreque, P. Hinrichsen y G. Cothran. 2005. Recuperación, conservación y caracterización del caballo chilote. Agro Sur, 33: 58-67.

Oltra, J., M. Ortiz, V. de la Barra y E. Stange. 1993. Tipificación de polimorfismos bioquímicos sanguíneos en equinos fina sangre de carrera y fina sangre chilena y su eficiencia en pruebas de exclusión de paternidad. Arch. Med. Vet., 25: 147-153.

Ott, J. 1992. Strategies for characterizing highly polymorphic markers in human gene mapping. Am. J. Hum. Genet., 51: 283-290.

Plante, Y., J.L. Vega-Pla, Z. Lucas, D. Colling, B. de March and F. Buchanan. 2007. Genetic diversity in a feral horse population from Sable Island, Canada. J. Hered., 98: 594-602.

Van Haeringen, H., A. Bowling, M. Stott, J. Lenstra and K. Zwaagstra. 1994. A highly polymorphic horse microsatellite locus VHL20. Anim. Genet., 25: 207.

Vega-Pla, J.L., J. Calderón, P. Rodríguez-Gallardo, A. Martínez and C. Rico. 2006. Saving feral horse populations: does it really matter? A case study of wild horses from Doñana National Park in southern Spain. Anim. Genet., 37: 571-578.

Raymond, M. and F. Rousset. 1995 a. An exact test for population differentiation. Evolution, 49: 12801283.

Raymond, M. and F. Rousset. 1995 b. GENEPOP 1.2: population genetics software for exact tests and ecumenicism. J. Hered., 86: 248-249.

Tautz, D. 1989. Hypervariability of simple sequences as a general source of polymorphic markers. Nucleic Acids Res., 17: 6463-6471.

Suenaga, E. and H. Nakamura. 2005. Evaluation of three methods for effective extraction of DNA from human hair. J. Chromatogr., 820: 137-141.

Waldbieser, G. and B. Bosworth. 1997. Cloning and characterization of microsatellite loci in chammel catfish, Ictalurus punctatus. Anim. Genet., 28: 295-298. 